\title{
Metastatic renal cell carcinoma regains sensitivity to tyrosine kinase inhibitor after nivolumab treatment: A case report
}

\author{
TAKESHI AZUMA ${ }^{1 *}$, TOHRU SUGIHARA $^{1 *}$, SACHI HONDA $^{1}$, \\ URAN YOSHIZAKI $^{1}$, FUSAKO NIIMI ${ }^{1}$, IBUKI TSURU ${ }^{1}$ and HARUKI KUME ${ }^{2}$ \\ ${ }^{1}$ Department of Urology, Tokyo Metropolitan Tama Medical Center, Fuchu, Tokyo 183-0042; ${ }^{2}$ Department of Urology, \\ The University of Tokyo Graduate School of Medicine, Tokyo 113-8654, Japan
}

Received September 2, 2018; Accepted February 4, 2019

DOI: $10.3892 / \mathrm{ol} .2019 .10027$

\begin{abstract}
Sequential therapy using tyrosine kinase inhibitors (TKIs) and mammalian target of rapamycin inhibitors is the mainstay of treatment for metastatic renal cell carcinoma. Recently, anti-programmed death-1 (PD-1) antibody, a type of immune checkpoint inhibitor, was approved for use against metastatic renal cell carcinoma. In the present report, two cases of TKI-refractory metastatic renal cell carcinoma which regained sensitivity to TKI after immunotherapy with nivolumab were described. In one case, a third challenge with axitinib after nivolumab treatment resulted in tumor shrinkage, although the second challenge with axitinib immediately before nivolumab treatment had no effect. In another case, a second challenge with pazopanib after nivolumab slightly reduced lung metastasis, which was refractory to pazopanib before nivolumab treatment. These cases suggest that nivolumab can influence the response to subsequent TKI treatment.
\end{abstract}

\section{Introduction}

Sequential therapy is the mainstay of treatment for metastatic renal cell carcinoma. Targeted therapy using tyrosine kinase inhibitors (TKIs) and mammalian target of rapamycin inhibitors has played an important role in sequential therapy (1). Inactivation of the von Hippel-Lindau (VHL) tumor suppressor protein is one characteristic of clear cell renal carcinoma. Loss of VHL gene is recognized in $60-70 \%$ of clear cell renal carcinoma $(2,3)$. Inactivation of VHL gene

Correspondence to: Dr Takeshi Azuma, Department of Urology, Tokyo Metropolitan Tama Medical Center, 2-8-29 Musashidai, Fuchu, Tokyo 183-0042, Japan

E-mail: tazuma-tky@umin.ac.jp

*Contributed equally

Key words: renal cell carcinoma, tyrosine kinase inhibitors, nivolumab, programmed death -1 , programmed death-ligand 1, immunotherapy, immunocheckpoint results in the up-regulation of hypoxia-inducible factor 1- $\alpha$ and the growth factor signaling, including vascular endothelial growth factor (VEGF), platelet-derived growth factor, and transforming growth factor-a. These factors play a key role in angiogenesis. Various multikinase inhibitors have been developed for the treatment of renal cancer (4).

Angiogenesis is the most important factor for the growing of renal cell carcinoma (RCC) (5). VEGF has been recognized as playing an important role in angiogenesis $(6,7)$. In RCC, high expression of VEGF and its receptor have been detected (8).

The two TKIs, axitinib and pazopanib, were approved for advanced RCC. VEGFR and PDGFR are presented as the main targets of both TKIs. Axitinib has a higher potency and selectivity against VEGFRs, especially VRGFR-2 $(9,10)$. Pazopanib targets other receptors, including C-kit, fibroblast growth factor receptors and colony-stimulating factor-1 receptor (11). Recently, the association between TKIs and tumor immunity has been reported. Both drugs can also augment antitumor immunity through reducing MDSCs which is one of immune suppressor cells $(12,13)$.

Recently, anti-programmed death-1 (PD-1) antibody, an immune checkpoint inhibitor (ICI), was approved for the treatment of metastatic renal cell carcinoma (14). PD-1 is expressed on T cells (15). Programmed death-1 ligand-1 (PD-L1) on cancer cells delivers an inhibitory signal to $\mathrm{T}$ cells through PD-1 (16). Anti-PD-1 antibody blocks the interaction between PD-L1 and PD-1, thereby activating the T cells against the cancer cells $(14,17)$. There was some difficulty initially in determining the best sequence for administering these drugs, with some studies suggesting that ICI might impact the response to subsequent cytotoxic agents $(18,19)$. In this report, we describe two cases in which sensitivity to TKIs was restored after immunotherapy with an anti-PD-1 antibody. These findings corroborate the hypothesis that ICI impacts the response to subsequently administered cytotoxic agents.

\section{Case report}

Case 1. A 56-year-old Japanese woman with multiple lung nodules on chest X-ray was referred to our hospital in 2012. Computed tomography (CT) disclosed bilateral renal tumors and bilateral adrenal, hepatic, lung, and lymph node metastases. Based on a biopsy of a renal tumor, RCC was diagnosed 
(cT3N2M1 stage IV). Her Karnofsky performance status was 100. The peripheral neutrophil and platelet counts, serum lactate dehydrogenase $(\mathrm{LDH})$, and corrected calcium $(\mathrm{cCa})$ values were normal. Based on both the Memorial Sloan-Kettering Cancer Center (MSKCC) and the International Metastatic RCC Database Consortium (IMDC) risk classifications, sequential targeted therapy was performed. She received five lines of targeted therapies from 2012 to 2016 in the following order: Sorafenib, sunitinib, temsirolimus, axitinib, and pazopanib. Each drug was stopped due to tumor progression on CT with the exception of pazopanib. Pazopanib was stopped due to the adverse effect of anemia. The administration period was 2, 2, 3, 36 and 3 months, respectively. The best response achieved was stable disease (SD), progressive disease (PD), PD, partial response (PR), and $\mathrm{SD}$, for sorafenib, sunitinib, temsirolimus, axitinib and pazopanib, respectively. At the end of the fifth line therapy, multiple lung and lymph node metastases had disappeared completely while the bilateral renal tumors and adrenal and hepatic metastases were still visible on CT.

As sixth line therapy, an axitinib rechallenge was administered in 2016 because this drug was the most effective among those previously used. Although the response after the second axitinib challenge was SD for eight months, the primary left renal tumor and bilateral adrenal metastases showed enlargement on CT (Fig. 1).

As the seventh line of therapy, immunotherapy with nivolumab was administered in 2017. After 16 administrations, the left renal tumor and bilateral adrenal metastases enlarged, and multiple lung metastases recurred (Fig. 1).

As the eighth line therapy, a third axitinib challenge was administered. One month later, the left renal tumor and multiple lung and bilateral adrenal metastases showed slight shrinkage. Surprisingly, six months later, multiple lung and bilateral adrenal metastases showed clear shrinkage (Fig. 1). Fourteen months after a third axitinib challenge, the metastases remained stable in their shrunken state.

Case 2. A 64-year-old Japanese man with a left renal tumor detected by ultrasonography was referred to our hospital in 2008. Based on the radiological findings, renal cell carcinoma was diagnosed (cT2N0M0, Stage II). He received a left radical nephrectomy. A pathological examination revealed clear cell carcinoma, Fuhrman Grade 2 and pT2. Three years later, multiple lung metastases were visible on CT. His Karnofsky performance status was 100 . The peripheral neutrophil and platelet counts and the serum $\mathrm{LDH}$ and $\mathrm{cCa}$ values were normal. Based on the MSKCC and IMDC risk classifications, sequential targeted therapy was performed. The patient received two lines of targeted therapy in the order of sunitinib and axitinib from 2011 to 2016. Each drug was stopped due to tumor progression on CT. The administration period was 24 and 36 months, and the best response achieved was SD for both sunitinib and axitinib.

After the second line therapy, CT showed that multiple lung, lymph node, pancreas, bone, and muscle metastases had developed (Fig. 2). As the third line therapy, pazopanib was administered in 2016. Three months later, the lung metastases slightly decreased in volume, and the response was SD. However, the lung, lymph node, and muscle metastases enlarged eight months later (Fig. 2).
As fourth line therapy, immunotherapy with nivolumab was administered in 2017. CT showed that after five administrations, new metastatic nodules had developed in the lung.

As fifth line therapy, a second pazopanib challenge was administered. Four months later, some of the lung metastases had shrunk, and the response was SD (Fig. 2). Eight months after a second pazopanib challenge, the metastases enlarged slightly from their shrunken state.

\section{Discussion}

We demonstrated two cases of metastatic TKI-refractory renal cell carcinoma which regained sensitivity to TKI after immunotherapy with nivolumab. Surprisingly, in Case 1, the third axitinib challenge after nivolumab caused dramatic tumor shrinkage although the second axitinib challenge immediately before nivolumab administration had no effect. The lack of $\mathrm{T}$ cell number of activation level in PMBCs for evaluation of ICI effect as a potential limitation of the study.

Recently, the hypothesis that ICI can impact the patient's response to subsequently cytotoxic agents was advanced $(18,19)$. In a phase III trial, nivolumab was able to improve overall survival to a greater extent than in the control but was unable to improve progression-free survival (20-22). Trials of a combination therapy with ICI and a cytotoxic agent have shown good results $(23,24)$. These studies revealed that a combination of ICI and cytotoxic agents had a synergistic effect. These findings are supported by the fact that the response to cytotoxic agents or targeted drugs improved after nivolumab therapy. Recently, some studies have reported findings supporting this hypothesis. In lung cancer, docetaxel after ICI had a stronger effect than docetaxel alone $(18,19)$. Albiges et al (25) also reported that in target therapy for RCC, ICI may impact the response to subsequent therapy. These reports suffered from the limitation of being retrospective. Our report, however, was able to corroborate this hypothesis.

Our findings strongly suggest that ICI affected cytotoxic agents and target drugs via the same mechanism. There are four possible explanations for the effectiveness of a rechallenge with a TKI after ICI: First, the presence of a heterogeneous cancer cell population (26) consisting of those sensitive to immunotherapy and those sensitive to TKI. Immunotherapy killed only the immunotherapy-sensitive cancer cells, leaving the TKI-sensitive cells to grow. Radiological examination apparently induced disease progression. Rechallenge with a TKI shrank the tumors consisting mostly of TKI-sensitive cells (Fig. 3); second, some patients treated with ICIs experienced an initial increase in the size of their tumors, confirmed by biopsy as inflammatory cell infiltrates or necrosis, which was followed by a decreased tumor burden. These delayed clinical responses were observed in patients with melanoma, sarcoma or bladder, breast, colorectal, esophageal, gastric, head and neck, lung, pancreatoduodenal, ovarian, renal cell or uterine cancer. In all these cases, an increase in the total tumor burden was later followed by tumor regression. These findings were classified as pseudoprogression merely due to nivolumab, and the rechallenge with the TKI was considered not to have had any effect, i.e., only a delayed response to ICI was observed $(27,28)$. Fujimoto have reported that $14(3 \%)$ patients with non-small cell lung cancer showed pseudoprogression of 542 patients 


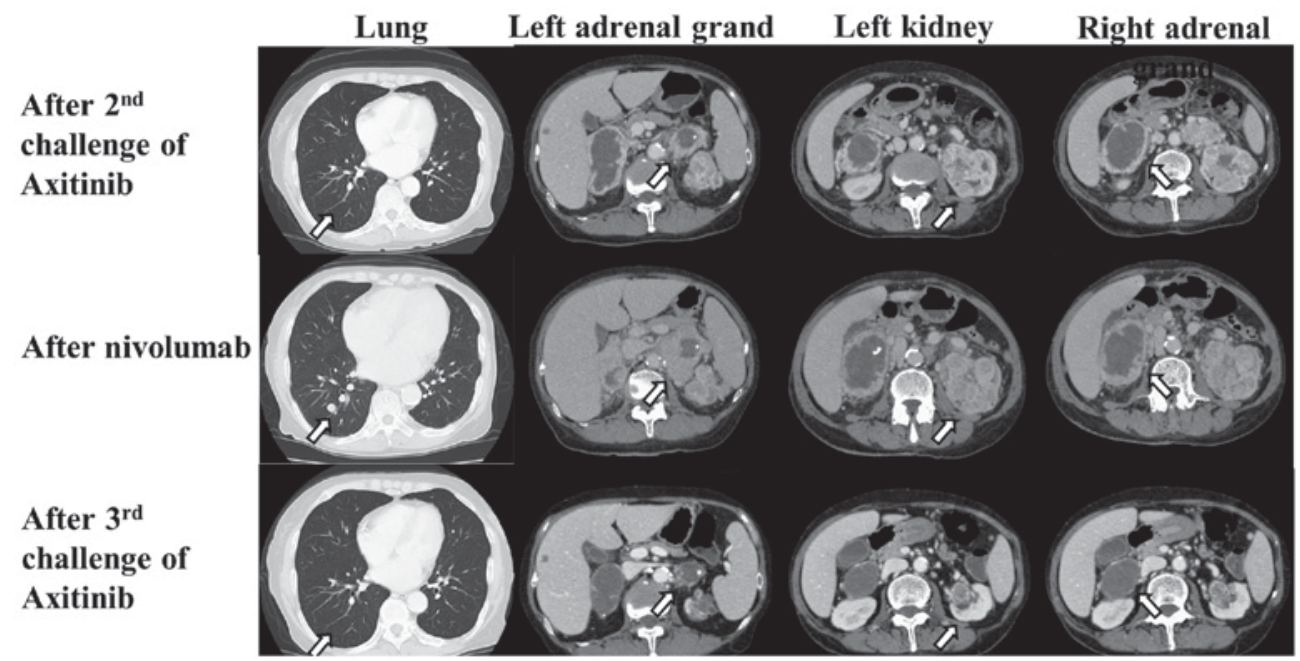

Figure 1. CT findings in Case 1. Chest CT showed the appearance of right lung metastasis during immunotherapy with nivolumab. The metastasis disappeared after the 3rd challenge with axitinib. Abominal CT showed an increase in the left primary renal tumor and bilateral adrenal metastasis during immunotherapy with nivolumab, which shrank following the 3rd challenge with axitinib. CT, computerized tomography; Rt, right; Lt, left.

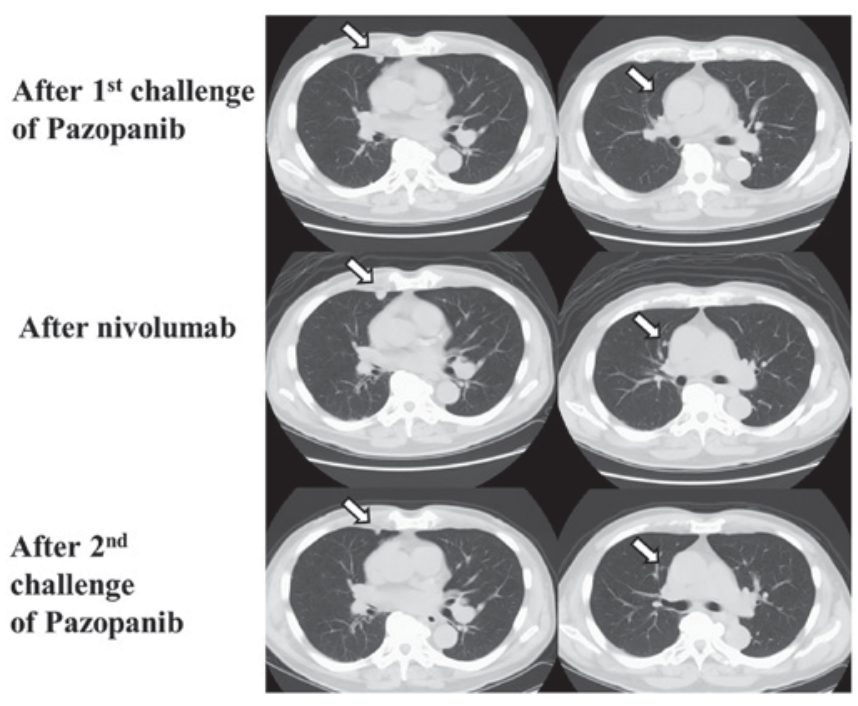

Figure 2. Chest CT findings in Case 2. Chest CT showed an increase in the lung metastases during immunotherapy with nivolumab, which shrank after the 2 nd challenge with pazopanib. CT, computerized tomography.

who received nivolumab monotherapy. Pseudoprogression was uncommon, and the response duration in patients with pseudoprogression was shorter than that in patients with typical response (29); third, the PD-L1 and PD-1 interaction was able to control T cell anergy, which the ICI reversed (30). The administration of ICI resolved the anergic state of the CTLs. These naïve CTLs were primed with antigens released from the tumor cells killed by the TKI (Fig. 4); and fourth, the administration of ICI resolved the anergic state of the CTLs, but these CTLs were suppressed by myeloid-derived suppressor cells (MDSCs). The CTLs were activated through the reduction of MDSCs by the TKI (31) (Fig. 5).

To conclude, sequential therapy is recommended for metastatic RCC (1). However, the optimal sequence of drug administration in sequential therapy is controversial. Recently, the effect of a combination therapy of ICI and a cytotoxic agent was tested with good results $(12,13)$. However, the optimal drug for combination with ICI is still uncertain. Clarifying the mechanism underlying this phenomenon is the first step to choosing the best drug and sequence of drug administration in sequential therapy.

\section{Acknowledgements}

Not applicable.

\section{Funding}

Data analysis and interpretation of this study was supported by JSPS KAKENHI Grant Number 17K11169 to T. Azuma.

\section{Availability of data and materials}

The datasets used and/or analysed during the current study are available from the corresponding author on reasonable request.

\section{Authors' contributions}

TA and HK collaborated in the conception and design of the study. TA, TS, SH, UY, FN, and IT collected the data and designed the analysis. TA analyzed the data. All authors were involved in writing the manuscript. All authors read and approved the final manuscript.

\section{Ethics approval and consent to participate}

Not applicable.

\section{Patient consent for publication}

All patients provided written informed consent for the publication of their data.

\section{Competing interests}

The authors declare that they have no competing interests. 
A

B

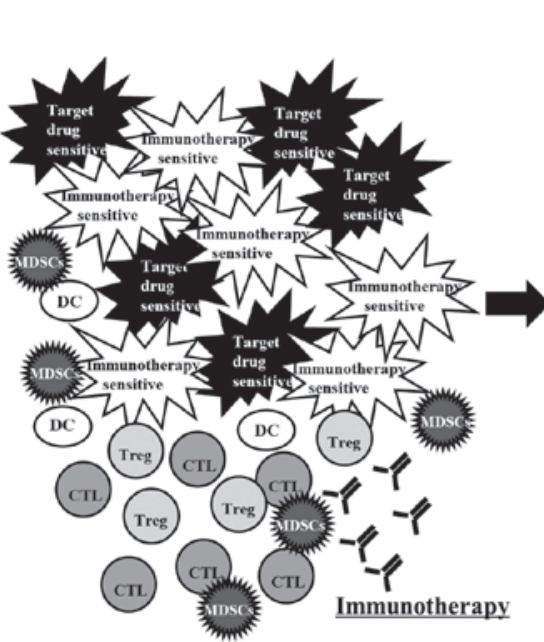

C
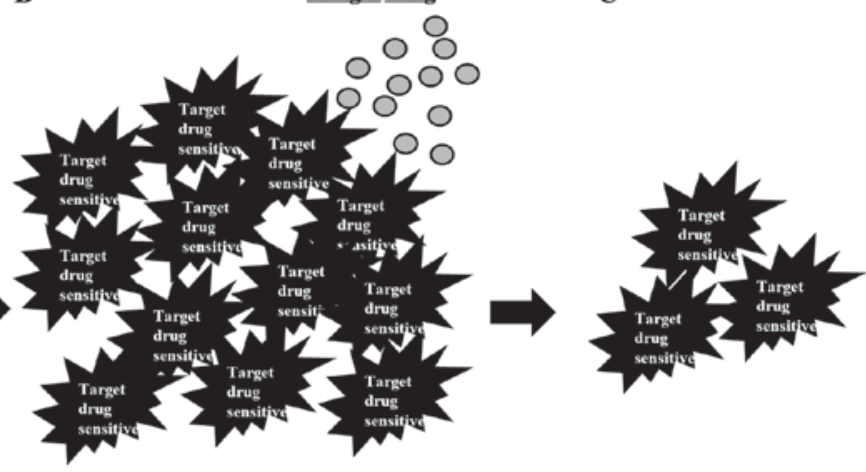

Figure 3. Hypothesis 1. (A) There are a heterogeneous cancer cell population consisting of those sensitive to immunotherapy and those sensitive to TKI. Immunotherapy destroyed only the immunotherapy-sensitive cancer cells. (B) The TKI-sensitive cells grow. Radiological examination apparently induced disease progression. (C) Rechallenge with a TKI shrank the tumors consisting mostly of TKI-sensitive cells. CTL, cytotoxic T lymphocyte; DC, dendritic cell; Treg, regulatory T cell; MDSCs, myeloid-derived suppressor cells; TKI, tyrosine kinase inhibitor.

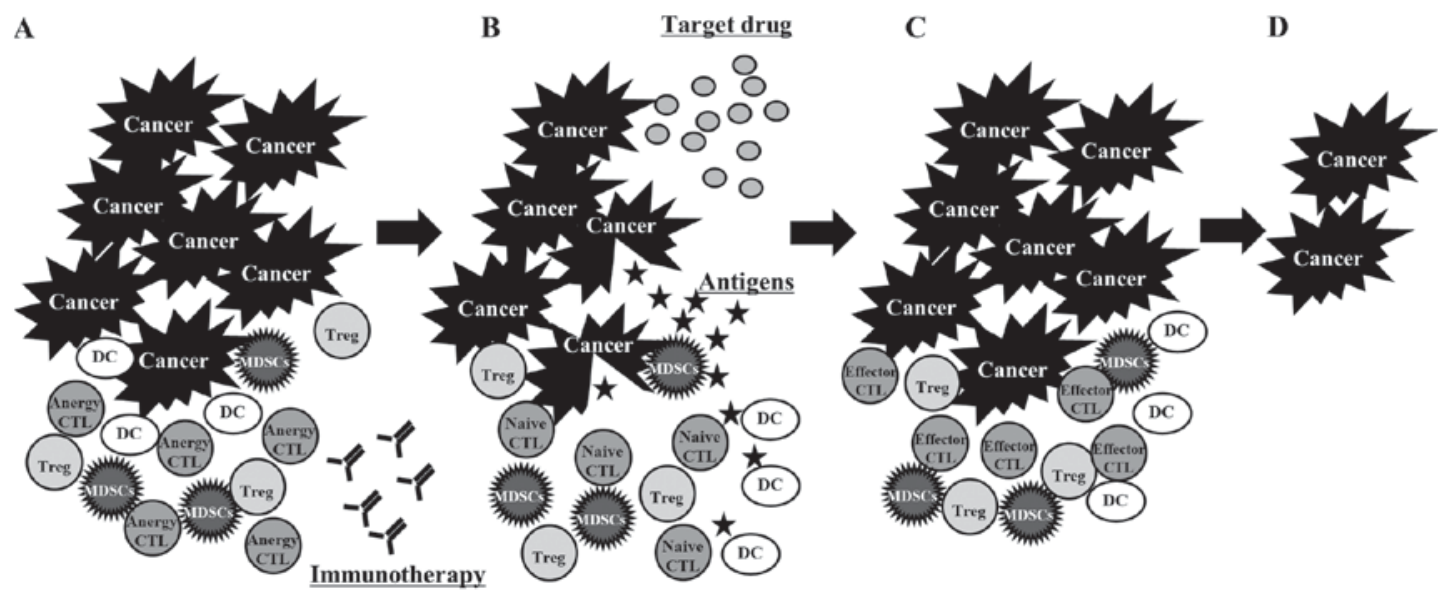

Figure 4. Hypothesis 3. (A) The PD-L1 and PD-1 interaction controls T cell anergy The administration of ICI resolved the anergic state of the CTLs. (B) The naïve CTLs were primed with antigens released from the tumor cells destroyed by the TKI. (C and D) The effector CTLs destroyed the cancer cells. CTL, cytotoxic T lymphocyte; DC, dendritic cell; Treg, regulatory T cell; MDSCs, myeloid-derived suppressor cells; ICI, immunocheckpoint inhibitor; TKI, tyrosine kinase inhibitor.

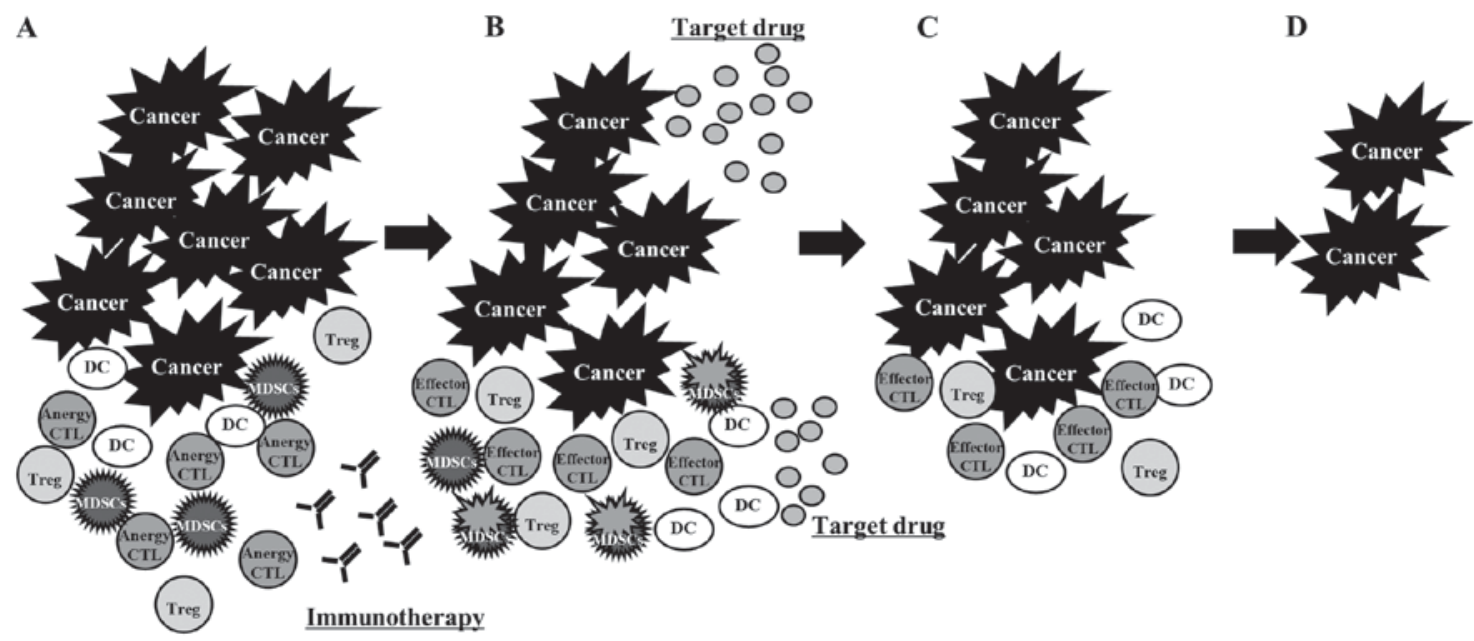

Figure 5. Hypothesis 4. (A) The administration of ICI resolved the anergic state of the CTLs $\rightarrow$. The effector CTLs were suppressed by MDSCs. (B) The CTLs were activated through the reduction of MDSCs by the TKI. (C and D) The effector CTLs destroyed the cancer cells. Cytotoxic T lymphocyte; DC, dendritic cell; Treg, regulatory T cell; MDSCs, myeloid-derived suppressor cells; ICI, immunocheckpoint inhibitor; TKI, tyrosine kinase inhibitor. 


\section{References}

1. Lambea J, Anido U, Etxániz O, Flores L, Montesa Á, Sepúlveda JM and Esteban E: The wide experience of the sequential therapy for patients with metastatic renal cell carcinoma. Curr Oncol Rep 18: 66, 2016.

2. Rathmell WK and Chen S: VHL inactivation in renal cell carcinoma: Implications for diagnosis, prognosis and treatment. Expert Rev Anticancer Ther 8: 63-73, 2008.

3. Gnarra JR, Tory K, Weng Y, Schmidt L, Wei MH, Li H, Latif F, Liu S, Chen F, Duh FM, et al: Mutations of the VHL tumour suppressor gene in renal carcinoma. Nat Genet 7: 85-90, 1994.

4. Cancer Genome Atlas Research Network: Comprehensive molecular characterization of clear cell renal cell carcinoma. Nature 499: 43-49, 2013.

5. Folkman J: The role of angiogenesis in tumor growth. Semin Cancer Biol 3: 65-71, 1992.

6. Ferrara $\mathrm{N}$ and Davis-Smyth T: The biology of vascular endothelial growth factor. Endocr Rev 18: 4-25, 1997.

7. Ferrara N, Gerber HP and LeCouter J: The biology of VEGF and its receptors. Nat Med 9: 669-676, 2003.

8. Tomisawa M, Tokunaga T, Oshika Y, Tsuchida T, Fukushima Y, Sato H, Kijima H, Yamazaki H, Ueyama Y, Tamaoki N and Nakamura M: Expression pattern of vascular endothelial growth factor isoform is closely correlated with tumour stage and vascularisation in renal cell carcinoma. Eur J Cancer 35: 133-137, 1999.

9. Bhargava P and Robinson MO: Development of second-generation VEGFR tyrosine kinase inhibitors: Current status. Curr Oncol Rep 13: 103-111, 2011.

10. Hu-Lowe DD, Zou HY, Grazzini ML, Hallin ME, Wickman GR, Amunds on K, Chen JH, Rewolinski DA, Yamazaki S, $\mathrm{Wu} \mathrm{EY}$, et al: Nonclinical antiangiogenesis and antitumor activities of axitinib (AG-013736), an oral, potent, and selective inhibitor of vascular endothelial growth factor receptor tyrosine kinases 1, 2, 3. Clin Cancer Res 14: 7272-7283, 2008.

11. Motzer RJ, Hutson TE, Cella D, Reeves J, Hawkins R, Guo J, Nathan P, Staehler M, de Souza P, Merchan JR, et al: Pazopanib versus sunitinib in metastatic renal-cell carcinoma. N Engl J Med 369: 722-731, 2013.

12. Yuan H, Cai P, Li Q, Wang W, Sun Y, Xu Q and Gu Y: Axitinib augments antitumor activity in renal cell carcinoma via STAT3-dependent reversal of myeloid-derived suppressor cell accumulation. Biomed Pharmacother 68: 751-756, 2014.

13. Pal SK, Hossain DM, Zhang Q, Frankel PH, Jones JO, Carmichael C, Ruel C, Lau C and Kortylewski M: Pazopanib as third line therapy for metastatic renal cell carcinoma: Clinical efficacy and temporal analysis of cytokine profile. J Urol 193: 1114-1121, 2015.

14. Topalian SL, Hodi FS, Brahmer JR, Gettinger SN, Smith DC, McDermott DF, Powderly JD, Carvajal RD, Sosman JA, Atkins MB, et al: Safety, activity, and immune correlates of anti-PD-1 antibody in cancer. N Engl J Med 366: 2443-2454, 2012.

15. Ishida Y, Agata Y, Shibahara K and Honjo T: Induced expression of PD-1, a novel member of the immunoglobulin gene superfamily, upon programmed cell death. EMBO J 11: 3887-3895, 1992.

16. Dong H, Zhu G, Tamada K and Chen L: B7-H1, a third member of the B7 family, co-stimulates T-cell proliferation and interleukin-10 secretion. Nat Med 5: 1365-1369, 1999.

17. Azuma T, Yao S, Zhu G, Flies AS, Flies SJ and Chen L: B7-H1 is a ubiquitous antiapoptotic receptor on cancer cells. Blood 111: 3635-3643, 2008.
18. Schvartsman G, Peng SA, Bis G, Lee JJ, Benveniste MFK, Zhang J, Roarty EB, Lacerda L, Swisher S, Heymach JV, et al: Response rates to single-agent chemotherapy after exposure to immune checkpoint inhibitors in advanced non-small cell lung cancer. Lung Cancer 112: 90-95, 2017.

19. Ogawara D, Soda H, Iwasaki K, Suyama T, Taniguchi H, Fukuda Y and Mukae H: Remarkable response of nivolumab-refractory lung cancer to salvage chemotherapy. Thorac Cancer 9: 175-180, 2018.

20. Motzer RJ, Escudier B, McDermott DF, George S, Hammers HJ, Srinivas S, Tykodi SS, Sosman JA, Procopio G, Plimack ER, et al: Nivolumab versus everolimus in advanced renal-cell carcinoma. N Engl J Med 373: 1803-1813, 2015.

21. Borghaei H, Paz-Ares L, Horn L, Spigel DR, Steins M, Ready NE, Chow LQ, Vokes EE, Felip E, Holgado E, et al: Nivolumab versus docetaxel in advanced nonsquamous non-small-cell lung cancer. N Engl J Med 373: 1627-1639, 2015.

22. Ferris RL, Blumenschein G Jr, Fayette J, Guigay J, Colevas AD, Licitra L, Harrington K, Kasper S, Vokes EE, Even C, et al: Nivolumab for recurrent squamous-cell carcinoma of the head and neck. N Engl J Med 375: 1856-1867, 2016.

23. Atkins MB, Plimack ER, Puzanov I, Fishman MN, McDermott DF, Cho DC, Vaishampayan U, George S, Olencki TE, Tarazi JC, et al: Axitinib in combination with pembrolizumab in patients with advanced renal cell cancer: A non-randomised, open-label, dose-finding, and dose-expansion phase $1 \mathrm{~b}$ trial. Lancet Oncol 19: 405-415, 2018.

24. Gandhi L, Rodríguez-Abreu D, Gadgeel S, Esteban E, Felip E, De Angelis F, Domine M, Clingan P, Hochmair MJ, Powell SF, et al: Pembrolizumab plus chemotherapy in metastatic non-small-cell lung cancer. N Engl J Med 378: 2078-2092, 2018.

25. Albiges L, Fay AP, Xie W, Krajewski K, McDermott DF, Heng DY, Dariane C, DeVelasco G, Lester R, Escudier B and Choueiri TK: Efficacy of targeted therapies after PD-1/PD-L1 blockade in metastatic renal cell carcinoma. Eur J Cancer 51: 2580-2586, 2015.

26. Fidler IJ: The pathogenesis of cancer metastasis: The 'seed and soil' hypothesis revisited. Nat Rev Cancer 3: 453-458, 2003.

27. Chen L, Azuma T, Yu W, Zheng X, Luo L and Chen L: B7-H1 maintains the polyclonal $\mathrm{T}$ cell response by protecting dendritic cells from cytotoxic T lymphocyte destruction. Proc Natl Acad Sci USA 115: 3126-3131, 2018.

28. Chiou VL and Burotto M: Pseudoprogression and immune-related response in solid tumors. J Clin Oncol 33: 3541-3543, 2015.

29. Fujimoto D, Yoshioka H, Kataoka Y, Morimoto T, Hata T, Kim YH, Tomii K, Ishida T, Hirabayashi M, Hara S, et al: Pseudoprogression in previously treated patients with non-small cell lung cancer who received nivolumab monotherapy. J Thorac Oncol: Nov 20, 2018 (Epub ahead of print).

30. Tsushima F, Yao S, Shin T, Flies A, Flies S, Xu H, Tamada K, Pardoll DM and Chen L: Interaction between B7-H1 and PD-1 determines initiation and reversal of T-cell anergy. Blood 110: 180-185, 2007.

31. Ko JS,Zea AH, Rini BI, Ireland JL,Elson P, Cohen P, Golshayan A, Rayman PA, Wood L, Garcia J, et al: Sunitinib mediates reversal of myeloid-derived suppressor cell accumulation in renal cell carcinoma patients. Clin Cancer Res 15: 2148-2157, 2009. 